
\title{
28 Research Square \\ sRAGE Downregulates the VEGF Expression in PCOS Ovarian Granulosa Cells
}

\section{Jingyan Wang}

Zhengzhou University Third Hospital and Henan Province Women and Children's Hospital

\section{Yichun Guan}

Zhengzhou University Third Hospital and Henan Province Women and Children's Hospital

Yi Liu

Fuwai Central China Cardiovascular Hospital

\section{Liang Wang}

Zhengzhou University Third Hospital and Henan Province Women and Children's Hospital

\section{Zhan Zhang}

Zhengzhou University Third Hospital and Henan Province Women and Children's Hospital

Bijun Wang ( $18738193657 @ 163 . c o m$ )

The Third Affiliated Hospital of Zhengzhou University

\section{Research}

Keywords: PCOS, sRAGE, VEGF, EGF-like growth factors, granulosa cells

Posted Date: May 11th, 2020

DOl: https://doi.org/10.21203/rs.3.rs-26984/v1

License: (c) (i) This work is licensed under a Creative Commons Attribution 4.0 International License.

Read Full License 


\section{Abstract}

Objective High expression of VEGF in ovarian tissue, serum and follicular fluid of PCOS women is involved in the physiological and pathogenesis processes of PCOS. Our objective was to investigate the effect of sRAGE on VEGF expression and EGF-like growth factor in PCOS ovarian granulosa cells.

Methods We collected ovarian granulosa cells of PCOS patients who underwent in vitro fertilization (IVF). Then treatment ovarian granulosa cells with different concentrations of sRAGE. Levels of VEGF, AREG, BTC and EREG mRNA were examined by quantitative RT-PCR. The protein levels of VEGF, AREG, BTC and EREG were measured by ELISA.

Results Treatment with sRAGE decrease the production of VEGF, and the effects were dependent on the concentrations of sRAGE $(P<0.05)$. Simultaneously, the expression of the EGF-like growth factors AREG, BTC and EREG were decreased, and the expression were dependent on the concentrations of sRAGE $(P<$ 0.05).

Conclusions SRAGE may downregulate VEGF expression in PCOS ovarian granulosa cells,and EGF-like growth factor pathway may be involved in this process.

\section{Introduction}

Polycystic ovary syndrome (PCOS) is a common endocrine and metabolic disorder syndrome in women of reproductive age, it affects about $6 \%$ of women of reproductive age ${ }^{[1]}$. Although, the pathogenesis of PCOS still remains unclear, studies suggest that the disorders in regulatory mechanisms of angiogenesis may play a role in pathogenesis of PCOS. Vascular endothelial growth factor (VEGF) is the most important member of family of angiogenic factors, which is expressed in the ovary and regulates angiogenesis in regular follicular growth, ovulation, and the subsequent development and regression of the corpus luteum ${ }^{[2]}$. Consistent with increased angiogenesis, several studies have demonstrated increased VEGF expression in ovarian tissue, serum and follicular fluid of PCOS women ${ }^{[3]}$. Taken together, these data suggest that VEGF is involved in the physiological and pathogenesis processes of PCOS by influencing the angiogenesis. Scientists have reached consensus and reported that inhibiting VEGF expression can improve ovarian function in women with PCOS [4].

The receptor for advanced glycation end products (RAGE) is a member of the immunoglobulin superfamily, which is involved in many pathological processes ${ }^{[5]}$. The soluble receptor for advanced glycation endproducts (SRAGE) is an extracellular form of RAGE that is missing the cytosolic and transmembrane domains. SRAGE acts as a decoy that interrupts adverse intracellular signaling caused by the AGE-RAGE axis and is not only a biological marker that reflects pathological changes, but is also a protective factor that delays the occurrence of many diseases ${ }^{[6]}$. Our previous study found that the follicular fluid SRAGE has a positive correlation with ovarian reservation and decreased in PCOS compared with women without PCOS ${ }^{[7]}$. One research supported our point that SRAGE could represent as 
a biomarker and a potential therapeutic target for ovarian dysfunction in PCOS ${ }^{[8]}$. Interestingly, we have found that follicular fluid sRAGE levels were negatively correlated with VEGF in PCOS ${ }^{[9]}$.

EGF-like growth factors, amphiregulin (AREG), betacellulin (BTC), epiregulin (EREG) can bind and activate epidermal growth factor receptor (EGFR) ${ }^{[10]}$. EGFR and its ligands are well known to be expressed in reproductive tissues and to mediate various female reproductive functions ${ }^{[11]}$, they participate in ovarian function through autocrine and (or) paracrine action. BTC play a role in VEGF regulation in head and neck squamous carcinoma cells (HNSCCs) ${ }^{[12]}$. AREG significantly increase VEGF production in the human granulosa cell line, $\mathrm{KGN}^{[13]}$. These results suggest that AREG, BTC, EREG mediate the production of VEGF. This year, there is also a research found that AREG-EGFR/HER2 partially mediates hCG-induced VEGF expression in human granulosa cells, suggesting that EGFR factor is the upstream factor of VEGF ${ }^{[14]}$.In the current study, we aimed to examine the effect of sRAGE on VEGF and EGF-like growth factors AREG, BTC, EREG expression in PCOS ovarian granulosa cells.

\section{Materials And Methods}

\section{Subjects}

The subjects in this study were recruited from patients who underwent in vitro fertilization-embryo transfer (IVF-ET) at our reproductive center. The present study included 10 patients with PCOS diagnosed according to the Rotterdam criteria ${ }^{[15]}$. All subjects in the study had no histories of genetic disease, immune infertility, ovarian surgery, uterine malformation and endocrine disease. Study was approved by the Ethics Committee of the third Affiliated Hospital of Zhengzhou University. All the subjects enrolled in the study gave written formal consent to participate.

\section{Experimental sample collection and determination}

Pituitary desensitization was initiated in midluteal phase by subcutaneous injection of a gonadotropinreleasing hormone agonist (GnRH-a, Decapeptyl, Ferring GmbH, Germany). Gonadotropin (Gn) stimulation was initiated after standard down regulation with human menopausal gonadotropin (hMG, Livzon, China) and recombinant FSH (Gonal-f, Merck, Germany) followed by human chorionic gonadotropin (hCG, Livzon, China) administration based on follicular size. Oocytes were collected by transvaginal ultrasound-guided puncture 34-36 h later.

\section{Collection and cultivation of granulosa cells}

Follicular fluid was centrifuged at $2000 \mathrm{r} / \mathrm{min}$ for $10 \mathrm{~min}$. Phosphate-buffered saline (PBS, $12 \mathrm{ml}$ from Hyclone, USA) was added to the cells to prepare a single-cell suspension. The suspension was added to a centrifuge tube containing hydroxypropylmethyl cellulose (Hao Yang Biological Formulation Company, Tianjin, China) in a 1:1 ratio, followed by centrifugation at $2000 \mathrm{r} / \mathrm{min}$ for $30 \mathrm{~min}$. PBS was added to the white cell layer in a 1:1 ratio, followed by centrifugation at $1000 \mathrm{r} / \mathrm{min}$ for $10 \mathrm{~min}$. The cells were placed in red cell lysis buffer and centrifuged at $1000 \mathrm{r} / \mathrm{min}$ at room temperature for one minute. After removal of 
the supernatant, the granulosa cells were cultured in a humidified atmosphere containing $95 \%$ air and $5 \%$ $\mathrm{CO}_{2}$ at $37^{\circ} \mathrm{C}$ in DMEM/F-12 medium supplemented with $10 \%$ charcoal $/$ dextran-treated $\mathrm{FBS}, 100 \mathrm{U} / \mathrm{ml}$ penicillin, $100 \mu \mathrm{g} / \mathrm{ml}$ streptomycin sulfate and 1X Gluta MAX. Cells were plated in 12-well plate at densities of $5 \times 10^{4} \mathrm{cells} / \mathrm{cm}^{2}$ with $1 \mathrm{ml}$ of culture medium. After 5 days, the medium was changed to medium containing $0.5 \% \mathrm{charcoal} /$ dextrantreated FBS. After another $48 \mathrm{~h}$, sRAGE was added at a concentration of $0,0.6,0.9$, or $1.2 \mu \mathrm{g} / \mathrm{mL}^{[16]}$. Granulosa cells cultured without sRAGE stimulation were used as the control group. After another $48 \mathrm{~h}$, quantitative real-time PCR and ELISA were used to detected mRNA and protein respectively.

\section{Reverse transcription quantitative real-time PCR}

Total RNA was isolated from the collected primary granulosa cells using Trizol reagent (Invitrogen, USA) according to the manufacturer's instructions. First-strand cDNA was synthesized from $2 \mu \mathrm{g}$ of total RNA by reverse transcriptase (Fermentas, Canada). The following primers were used for reverse transcription quantitative real-time PCR(RT-qPCR): AREG, 5'- TAG AGC ACC TGG AAG CAG TAA C -3' (Forward primer) and 5'- CCT GAC GTA TTG TCT TCT AAG - 3' (Reverse primer); BTC, 5'- TGC CCT GGG TCT AGT GAT CCT TC -3' (Forward primer) and 5'- GTG GCC TTT CCG CTT TGA TTG TG -3' (Reverse primer); EREG, 5'- GTT TCC ATC TTC TAC AGG CAG TC -3' (Forward primer) and 5'- GCA AAC AAT AGC CAT TCA TGT CAG A -3' (Reverse primer); VEGF, 5'- ATC CAA TCG AGA CCC TGG TG -3' (Forward primer) and 5'- ATC TCT CCT ATG TGC TGG CC -3' (Reverse primer) and $\beta$-actin, 5'- CAC GAT GGA GGG GCC GGA CTC ATC - 3' (Forward primer) and 5'- TAA AGA CCT CTA TGC CAA CAC AGT - 3' (Reverse primer). The mRNA levels of ARGE, BTC, EREG and VEGF were determined by RT-PCR (EDC-810, EASTWIN, China). The CDNA was then amplified in triplicate using SYBR Green/Fluorescein PCR master mix (Fermentas, Canada) and detected on an ABI Prism 7900 Sequence PCR machine (Illumina, USA). All RT-PCR experiments were performed in triplicate, and the mean value was used to determine the mRNA levels. Water and mRNA without reverse

transcriptase were used as negative controls. The levels of mRNA relative to $\beta$-actin were calculated using the $2^{-\triangle \Delta \mathrm{Ct}}$ cycle threshold method. All primers were provided by GenScript Company (China branch).

\section{Enzyme-linked immunosorbent assay}

The fluid from the first large aspirated follicle without blood was collected for ELISA assessments of VEGF, ARGE, BTC and EREG: Quantikine Human VEGF Immunoassay, Quantikine Human ARGE mmunoassay, Quantikine Human BTC Immunoassay, and Quantikine Human EREG Immunoassay (R\&D Systems, Minneapolis, MN, USA). The results are given in picograms per milliliter ( $\mathrm{pg} / \mathrm{mL})$.

\section{Statistical analysis}

Statistical analysis was performed using SPSS software (version 17.0 for Windows; SPSS Inc., Chicago, $\mathrm{IL}$ ). The means and standard deviations (mean $\pm \mathrm{SD}$ ) was used to describe variables. Multiple comparisons were analyzed by one-way ANOVA followed by LSD-t multiple comparison tests. A significant difference was defined as $P \otimes 0.05$. 


\section{Exogenous SRAGE decreases VEGF expression in ovarian granulosa cells}

The VEGF mRNA and protein were detected by RT-qPCR and ELISA, respectively. After treating granulosa cells with different concentrations of sRAGE $(0.6 \mu \mathrm{g} / \mathrm{ml}, 0.9 \mu \mathrm{g} / \mathrm{ml}$, or $1.2 \mu \mathrm{g} / \mathrm{ml})$, VEGF mRNA and protein levels were both lower compared to untreated granulosa cells, and the effects of sRAGE on VEGF expression were dose-dependent (Fig. 1).

\section{Exogenous sRAGE decreases ARGE, BTC and EREG expression in ovarian granulosa cells}

The EGF-like growth factors ARGE, BTC and EREG mRNA and protein were detected by RT-qPCR and ELISA, respectively. After treatment with different concentrations of sRAGE $(0.6 \mu \mathrm{g} / \mathrm{ml}, 0.9 \mu \mathrm{g} / \mathrm{ml}$, or $1.2 \mu \mathrm{g} / \mathrm{ml})$, ARGE, BTC and EREG mRNA and protein levels were both lower compared to untreated granulosa cells, and the effects of sRAGE on ARGE, BTC and EREG expression were dose-dependent (Fig. 2).

\section{Discussion}

In the present study, we treatment PCOS ovarian granulosa cells with difference concentration of sRAGE, and examined the expression of VEGF and EGF-like growth factors AREG, BTC and EREG. Our results show that SRAGE decrease the production of VEGF and EGF-like growth factors AREG, BTC and EREG, and the effects were dependent on the concentrations of SRAGE. It demonstrated that SRAGE may downregulate VEGF expression via EGF-like growth factor pathway in PCOS ovarian granulosa cells and SRAGE may play a potential protective role in polycystic ovary syndrome.

AGE-RAGE axis can activate p21 RAS, MAPK (p38), PI3K, NF-kB and other cell signaling molecules, contribute to angiogenesis, apoptosis and inflammatory response processes ${ }^{[17]}$. In PCOS ovaries, AGEs and RAGE were stronger displayed in the granulosa cell layer compared to healthy ovaries ${ }^{[18]}$. To date, emerging evidences have supported that PCOS was related to hyperandrogenism, insulin resistance and chronic inflammation ${ }^{[19]}$. Our previous study shown that SRAGE concentrations were decreased in the follicular fluid of PCOS patients, and these sRAGE were inversely associated with VEGF, TNF-a, IL-6, and CRP protein levels ${ }^{[9]}$. According to Boulanger, the activation of AGEs-RAGE axis in human peritoneal mesothelial cells can induce the release of VEGF and subsequently the formation of capillary tubes, while anti-receptor for AGEs (RAGE) antibody reduced capillary tube formation ${ }^{[20]}$. As a decoy receptor for AGEs, sRAGE can block multiple cell signaling pathways induced by AGE-RAGE aixs. Psoriasin (S100A7) increases the expression of VEGF through the RAGE pathway and promote endothelial cell proliferation, however treatment with SRAGE inhibited endothelial cell proliferation and tube formation enhanced by recombinant psoriasin ${ }^{[21]}$. In the present study, we demonstrated that sRAGE reduced the expression of 
VEGF mRNA and protein in a dose-dependent manner in PCOS granulosa cells. Culturing human esophageal cancer cells with the administration of $0.2 \mathrm{ug} / \mathrm{ml}$ sRAGE for $24 \mathrm{~h}$, sRAGE significantly inhibit the proliferation of esophageal cancer cells and VEGF-C expression ${ }^{[22]}$. Moreover, Emman found that treatmeng human umbilical vein Vascular endothelial cells (HUVECs) with $50 \mathrm{ng} / \mathrm{ml}$ sRAGE for 30 minutes, the amount of VEGF mRNA was significantly reduced ${ }^{[21]}$.

EGF-like growth factor AREG, BTC and EREG is the upstream regulators of VEGF, regulate the production of VEGF. Recombinant human AREG has been shown stimulated FLS to proliferate and produce VEGF in a dose-dependent manner in patients with rheumatoid arthritis ${ }^{[23]}$. In addition, BTC upregulate VEGF expression by activation of EGFR in HNSCCs, it also demonstrated that EGFR and c-erbB-2 signaling pathway(s) plays a role in VEGF regulation in $\mathrm{HNSCCs}^{[13]}$. In the female reproductive system, EGF-like growth factor and its receptor EGFR is widely expressed in ovarian tissue, and involved in female reproductive function ${ }^{[12]}$. In the menstrual cycle, LH peak induce EGF-like growth factor AREG, BTC and EREG transient increase, and these three factors have LH-like effect, inhibite AREG, BTC and EREG pathway can block LH signaling pathway ${ }^{[24]}$. As we all know, LH peak can promote the expression of VEGF in theca cells and luteinized granulosa cells ${ }^{[25]}$. AREG significantly increase VEGF production in the human granulosa cell line, KGN ${ }^{[14]}$.

In the present study, we found that sRAGE reduced the expression of AREG, BTC and EREG in a dosedependent manner in PCOS ovarian granulosa cells. Many studies have shown that SRAGE inhibits inflammatory responses and oxidative stress. Moreover, overexpression of soluble RAGE in mesenchymal stem cells enhances their immunoregulatory potential for cellular therapy in autoimmune arthritis, indicating that SRAGE has no toxicity to cells ${ }^{[26]}$. Taken together, sRAGE reduced EGF-like growth factors AREG, BTC and EREG expression while simultaneously reducing VEGF production in PCOS ovarian granulosa cells, as AREG, BTC and EREG are upstream regulators of VEGF, so these results demonstrated that SRAGE downregulates VEGF expression via EGF-like growth factor pathway in PCOS ovarian granulosa cells. The mechanisms underlying the effects of sRAGE on EGF-like growth factor and VEGF expression involve specific factor-mediated signaling pathways. In immortalized human granulosa cells, SVOG, treatment with AREG, BTC, or EREG upregulated COX-2 expression, the AREG-, BTC-, and EREGinduced COX-2 expression in turn contributed to PGE2 production ${ }^{[27]}$. The expression PGE2 and VEGF are closely related, both of which are important factors for regulating angiogenesis and vascular permeability. In human granulosa cells PGE2 can promote the expression of VEGF, involved in the regulation of angiogenesis of the pathological ovary ${ }^{[28]}$. AGE-bovine serum albumin increased production of COX-2 and PGE2, while sRAGE reduced AGE-stimulated COX-2 and PGE2 ${ }^{[29]}$. Taken together, these evidences implicating the involvement of PGE2 in the regulation of VEGF expression mediated by sRAGE. So far did not see the reports that whether SRAGE could reduce influence PGE2 synthesis through AREG, BTC and EREG, subsequently inhibiting the production of VEGF in PCOS granulosa cells, it is necessary to further study. 
In conclusion, sRAGE may downregulate VEGF expression in PCOS ovarian granulosa cells,and EGF-like growth factor pathway may be involved in this process.

From the new perspective of SRAGE, we want to provide further new ideas for the prevention and treatment of PCOS.

\section{List Of Abbreviations}

PCOS polycystic ovarian syndrome

AGEs advanced glycation end products

RAGE receptor for advanced glycation end products

sRAGE solube isoform of the receptor for AGEs

VEGF vascular endothelial growth factor

EGF epidermal growth factor receptor

AREG amphiregulin

BTC betacellulin

EREG epiregulin

TNF-a, tumour necrosis factor-a

IL-6 interleukelin-6

CRP C-reactive protein

IVF-ET in vitro fertilization-embryo transfer

\section{Declarations}

\section{Ethics approval and consent to participate}

This study was approved by the Ethics Committee of Third Affiliated Hospital of Zhengzhou University.

Consent for publication

All authors have read and approved the final version of the manuscript

\section{Availability of data and materia}


The dataset supporting the conclusions of this article is included within the Article.

\section{Competing interests}

The authors declare that they have no competing interests.

\section{Authors' contributions}

Jingyan Wang, Bijun Wang and Zhan Zhang contributed to the study design, experiment and manuscript preparation; Yichun Guan and Liang Wang were responsible for collecting samples, Yi Liu handled patient recruitment and statistical work. All authors read and approved the final manuscript.

\section{Funding}

This work was supported by grant 17020320701 from the Special fund for clinical research of the Chinese Medical Association: Protective effects and molecular mechanism of sRAGE on inhibiting inflammation and improving insulin resistance in PCOS.

\section{Acknowledgments}

The language corrections in this study were made with the help of American Journal Experts (AJE).

\section{References}

1. Goodarzi MO, Dumesic DA, Chazenbalk G, Azziz R. Polycystic ovary syndrome: etiology, pathogenesis and diagnosis[J]. Nat Rev Endocrinol. 2011;7(4):219-31.

2. Sugino N, Suzuki T, Sakata A, Miwa I, Asada H, Taketani T, et al. Angiogenesis in the human corpus luteum: changes in expression of angiopoietins in the corpus luteum throughout the menstrual cycle and in early pregnancy[J]. J Clin Endocrinol Metab. 2005;90(11):6141-8.

3. Fraser HM. Regulation of the ovarian follicular vasculature[J]. 4. Reproductive biology: RB\&E; 2006. p. 18.

4. Abramovich D, Irusta G, Bas D, Cataldi NI, Parborell F, Tesone M. Angiopoietins/TIE2 system and VEGF are involved in ovarian function in a DHEA rat model of polycystic ovary syndrome[J]. Endocrinology. 2012;153(7):3446-56.

5. Palimeri S, Palioura E, Diamanti-Kandarakis E. Current perspectives on the health risks associated with the consumption of advanced glycation end products: recommendations for dietary management[J]. Diabetes Metab Syndr Obes. 2015;8:415-26.

6. Lazo M, Halushka MK, Shen L, Maruthur N, Rebholz CM, Rawlings AM, et al. Soluble receptor for advanced glycation end products and the risk for incident heart failure: The Atherosclerosis Risk in Communities Study[J]. Am Heart J. 2015;170(5):961-7.

7. Wang B, Li J, Yang Q, Zhang F, Hao M, Guo Y. Decreased levels of sRAGE in follicular fluid from patients with PCOS[J]. Reproduction. 2017;153(3):285-92. 
8. Garg D, Grazi R, Lambert-Messerlian GM, Merhi Z. Correlation between follicular fluid levels of sRAGE and vitamin D in women with PCOS[J]. J Assist Reprod Genet. 2017;34(11):1507-13.

9. Wang B, Hao M, Yang Q, Li J, Guo Y. Follicular fluid soluble receptor for advanced glycation endproducts (sRAGE): a potential protective role in polycystic ovary syndrome[J]. J Assist Reprod Genet. 2016;33(7):959-65.

10. Harris RC, Chung E, Coffey RJ. EGF receptor ligands[J]. Exp Cell Res. 2003;284(1):2-13.

11. Schneider MR, Wolf $E$. The epidermal growth factor receptor and its ligands in female reproduction: insights from rodent models[J]. Cytokine Growth Factor Rev. 2008;19(2):173-81.

12. Oc P, Rhys-Evans $P$, Modjtahedi $H$, Eccles SA. Vascular endothelial growth factor family members are differentially regulated by c-erbB signaling in head and neck squamous carcinoma cells[J]. Clin Exp Metastasis. 2000;18(2):155-61.

13. Karakida S, Kawano Y, Utsunomiya Y, Furukawa Y, Sasaki T, Narahara H. Effect of heparin-binding EGF-like growth factor and amphiregulin on the MAP kinase-induced production of vascular endothelial growth factor by human granulosa cells[J]. Growth Factors. 2011;29(6):271-7.

14. Lanlan Fang Y, Yu Y, Li S, Wang J, He, et al. Upregulation of AREG, EGFR, and HER2 contributes to increased VEGF expression in granulosa cells of patients with OHSS[J]. Biology of reproduction.2019,0(0),1-7.

15. Rotterdam EA-SPcwg. Revised 2003 consensus on diagnostic criteria and long-term health risks related to polycystic ovary syndrome (PCOS)[J]. Hum Reprod. 2004;19(1):41-7.

16. Wang B-J, Qian L, Li J, Wang F, Yang Q-L, et al. sRAGE plays a role as a protective factor in the development of PCOS by inhibiting inflammation[J].2019 Jun 28:1-4.

17. Yamagishi S. Role of advanced glycation end products (AGEs) and receptor for AGEs (RAGE) in vascular damage in diabetes[J]. Exp Gerontol. 2011;46(4):217-24.

18. Diamanti-Kandarakis E, Piperi C, Patsouris E, Korkolopoulou P, Panidis D, Pawelczyk L, et al. Immunohistochemical localization of advanced glycation end-products (AGEs) and their receptor (RAGE) in polycystic and normal ovaries[J]. Histochem Cell Biol. 2007;127(6):581-9.

19. Qiao J, Feng HL. Extra- and intra-ovarian factors in polycystic ovary syndrome: impact on oocyte maturation and embryo developmental competence[J]. Hum Reprod Update. 2011;17(1):17-33.

20. Boulanger E, Grossin N, Wautier MP, Taamma R, Wautier JL. Mesothelial RAGE activation by AGEs enhances VEGF release and potentiates capillary tube formation[J]. Kidney Int. 2007;71(2):126-33.

21. Shubbar E, Vegfors J, Carlstrom M, Petersson S, Enerback C. Psoriasin. (S100A7) increases the expression of ROS and VEGF and acts through RAGE to promote endothelial cell proliferation[J]. Breast Cancer Res Treat. 2012;134(1):71-80.

22. Chen CG, Tang P, Yu ZT. [Effect of HMGB1 on the VEGF-C expression and proliferation of esophageal squamous cancer cells][J]. Zhonghua Zhong Liu Za Zhi. 2012;34(8):566-70.

23. Yamane S, Ishida S, Hanamoto Y, Kumagai K, Masuda R, Tanaka K, et al. Proinflammatory role of amphiregulin, an epidermal growth factor family member whose expression is augmented in 
rheumatoid arthritis patients[J]. J Inflamm (Lond). 2008;5:5.

24. Dahlhoff M, Wolf E, Schneider MR. The ABC of BTC: structural properties and biological roles of betacellulin[J]. Semin Cell Dev Biol. 2014;28:42-8.

25. Kamat BR, Brown LF, Manseau EJ, Senger DR, Dvorak HF. Expression of vascular permeability factor/vascular endothelial growth factor by human granulosa and theca lutein cells. Role in corpus luteum development[J]. Am J Pathol. 1995;146(1):157-65.

26. Park MJ, Lee SH, Moon SJ, Lee JA, Lee EJ, et al. Overexpression of soluble RAGE in mesenchymal stem cells enhances their immunoregulatory potential for cellular therapy in autoimmune arthritis.Sci Rep[J]. 2016 Nov 2; 6:35933.

27. Fang L, Cheng JC, Chang HM, Sun YP, Leung PC. EGF-like growth factors induce COX-2-derived PGE2 production through ERK $1 / 2$ in human granulosa cells[J]. J Clin Endocrinol Metab. 2013;98(12):4932-41.

28. Duncan WC, Nio-Kobayashi J. Targeting angiogenesis in the pathological ovary[J]. Reprod Fertil Dev. 2013;25(2):362-71.

29. Nah SS, Choi IY, Lee CK, Oh JS, Kim YG, Moon HB, et al. Effects of advanced glycation end products on the expression of COX-2, PGE2 and NO in human osteoarthritic chondrocytes[J]. Rheumatology. 2008;47(4):425-31.

\section{Figures}

A

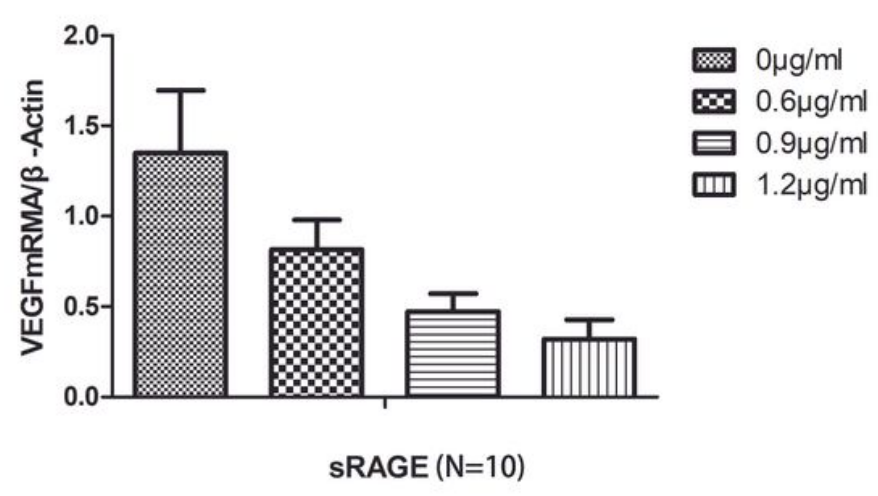

B

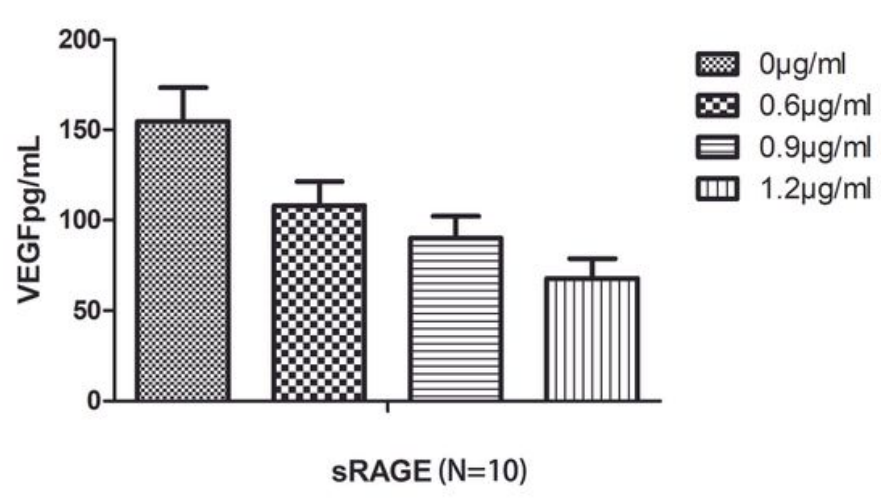

\section{Figure 1}

The direct effects of SRAGE on the expression and secretion of VEGF in ovarian granulosa cells isolated from patients with polycystic ovarian syndrome (PCOS). (A) VEGF mRNA levels were decreased by approximately $0.2-, 0.5$-and 0.8 -fold in granulosa cells treated with $0.6,0.9$, and $1.2 \mu \mathrm{g} / \mathrm{mL}$ sRAGE, respectively, compared with untreated granulosa cells $(1.349 \pm 0.347 \otimes 0.817 \pm 0.163 \varangle 0.472 \pm 0.101 \mathrm{vs}$ $0.321 \pm 0.106, F=24.70, P \otimes 0.05)$. The differences between each pair of groups were signifcant $(P<0.05)$. (C) 
VEGF protein levels were decreased by approximately $0.3-, 0.7$-, and 0.8 -fold in granulosa cells treated with $0.6,0.9$, and $1.2 \mu \mathrm{g} / \mathrm{mL}$ sRAGE, respectively, compared with untreated granulosa cells $(154.821 \pm 18.653,108.194 \pm 13.196,90.123 \pm 12.137 v s 67.948 \pm 11.033, F=68.902, P \otimes 0.05)$. The differences between each pair of groups were signifcant $(P<0.05)$. VEGF mRNA and protein levels were detected by RT-PCR and ELISA, respectively. SRAGE, soluble receptor for advanced glycation end products; VEGF, vascular endothelial growth factor.

A

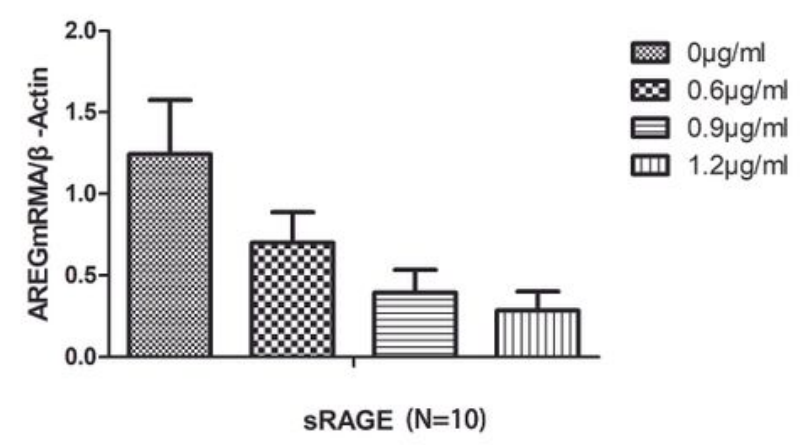

C
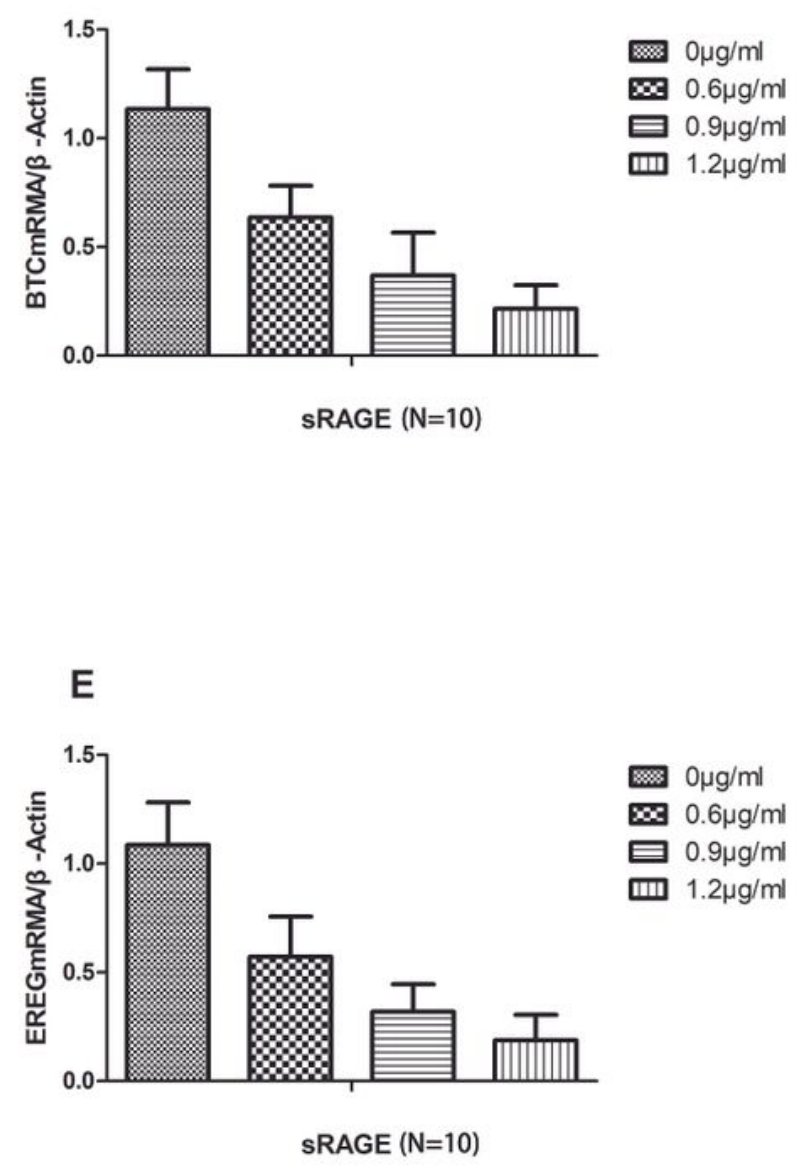

\section{B}

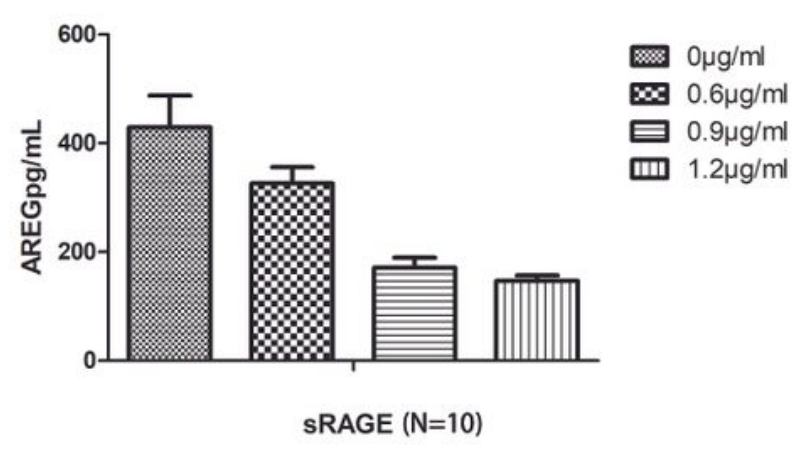

D

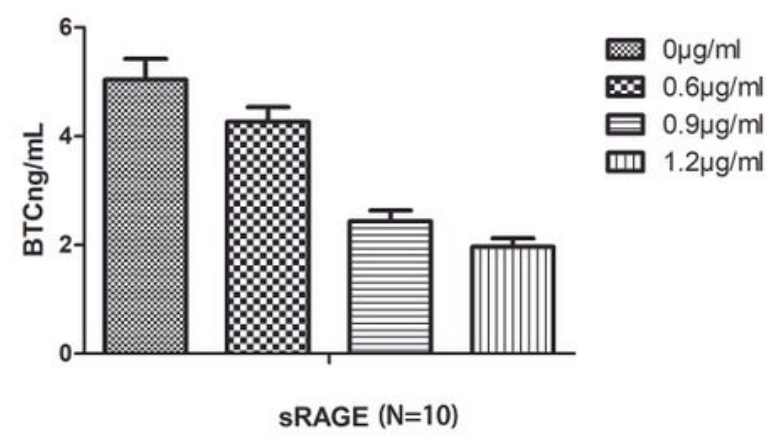

$\mathbf{F}$

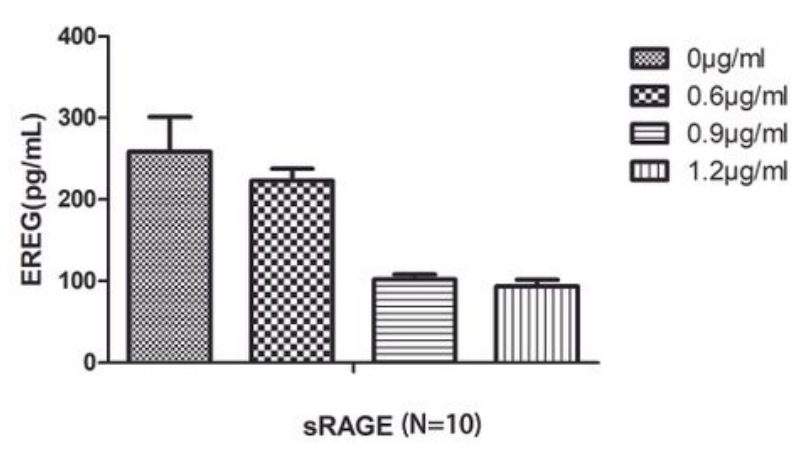

Figure 2 
The effect of the EGF-like growth factor signal pathway on VEGF induction by sRAGE. (A) ARGE mRNA levels were decreased in granulosa cells treated with $0.6,0.9$, and $1.2 \mu \mathrm{g} / \mathrm{mL}$ sRAGE, compared with untreated granulosa cells $(1.244 \pm 0.330,0.701 \pm 0.187,0.394 \pm 0.141$ vs $0.284 \pm 0.117, F=20.84 \llbracket P \llbracket 0.05)$. (B) ARGE levels were decreased in granulosa cells treated with $0.6,0.9$, and $1.2 \mu \mathrm{g} / \mathrm{mL}$ sRAGE, compared with untreated granulosa cells $(429.09 \pm 58.16,326.11 \pm 30.27,171.47 \pm 17.98$ vs $146.67 \pm 10.00, F=150.69 \mathrm{PP} \nabla$ 0.05). (C) BTC mRNA levels were decreased in granulosa cells treated with $0.6,0.9$, and $1.2 \mu \mathrm{g} / \mathrm{mL}$ sRAGE, compared with untreated granulosa cells $(1.133 \pm 0.184 \otimes 0.636 \pm 0.146 \rrbracket 0.369 \pm 0.197$ vs $0.215 \pm 0.109$, $F=30.61 \otimes P \otimes 0.05)$. (D) BTC levels were decreased in granulosa cells treated with $0.6,0.9$, and $1.2 \mu \mathrm{g} / \mathrm{mL}$ sRAGE, compared with untreated granulosa cells $(5.04 \pm 0.38,4.26 \pm 0.27,2.43 \pm 0.20$ vs $1.96 \pm 0.16$, $F=306.12 \varangle P \llbracket 0.05$ ). (E) EREG mRNA levels were decreased in granulosa cells treated with 0.6, 0.9, and $1.2 \mu \mathrm{g} / \mathrm{mL}$ sRAGE, compared with untreated granulosa cells $(1.086 \pm 0.196 \rrbracket 0.572 \pm 0.184 \llbracket 0.320 \pm 0.125 \mathrm{vs}$ $0.187 \pm 0.117, F=31.06 \varangle P \llbracket 0.05$ ). (F) EREG levels were decreased in granulosa cells treated with $0.6,0.9$, and $1.2 \mu \mathrm{g} / \mathrm{mL}$ sRAGE, compared with untreated granulosa cells $(258.41 \pm 42.74,222.98 \pm 14.92$, $102.03 \pm 6.14$ vs $93.36 \pm 8.32, F=130.55 \llbracket P \otimes 0.05)$. ARGE, $B T C$ and EREG mRNA and protein levels were detected by RT-PCR and ELISA, respectively. SRAGE, soluble receptor for advanced glycation end products; ARGE, amphiregulin; BTC, betacellulin; EREG, epiregulin. 